\title{
基于[3+2]环加成反应的氮杂螺[3,4]环辛烷的合成
}

\author{
王雯陆秀宏董肖椿* 赵伟利* \\ (复旦大学药学院 上海 201203)
}

\begin{abstract}
摘要 哌嗪、吗啉是小分子药物中常见的结构片段, 2,6-二氮杂螺 $[3,4]$ 环辛烷和 2-氧-6-氮杂螺 [3,4]环辛烷等四元螺环化 合物作为其类似物, 在药物化学领域具有良好的应用前景. 本研究以廉价易得的丙二酸二乙酯或丙烯酸乙酯为起始原 料, 经 [3+2]环加成反应，可便捷高效地完成 6-芐基-2,6-二氮杂螺 $[3,4]$ 环辛烷和 2-氧-6-氮杂螺 $[3,4]$ 环辛烷克级水平的 合成.

关键词 2,6-二氮杂螺[3,4]环辛烷; 2-氧-6-氮杂螺[3,4]环辛烷; [3+2]环加成
\end{abstract}

\section{Synthesis of Azaspiro[3.4]octanes via [3+2] Cycloaddition}

\author{
Wang, Wen Lu, Xiuhong Dong, Xiaochun* Zhao, Weili* \\ (School of Pharmacy, Fudan University, Shanghai 201203)
}

\begin{abstract}
Piperazine and morpholine are common modules in drugs. Design and synthesis of their surrogates may help to explore the chemical and patent space in medicinal chemistry. In this article, through [3+2] cycloaddition, the improved synthesis of 6-benzyl-2,6-diazaspiro[3.4] octane oxalate and 2-oxa-6-azaspiro[3.4]octane as substitutes of piperazine and morpholine was provided, respectively. Multi-gram quantities of the compounds could be easily obtained in relatively high yields.

Keywords 2,6-diazaspiro[3,4]octane; 2-oxa-6-azaspiro[3,4]octane; [3+2] cycloaddition
\end{abstract}

吗啉、哌嗪等杂环是小分子化学药物中常见的结构 片段 ${ }^{[1]}$. 截止至 2012 年, 在美国食品药品监督管理局 (FDA) 已批准上市的 1552 种小分子药物中, 分别有 178 种包含哌嗪环、83 种包含吗啉环 ${ }^{[2]}$. 自 2006 年起, Carreira 等 ${ }^{[3]}$ 提出将四元螺环类化合物作为六元杂环的 类似物, 并将其用于小分子药物的结构改造, 为药物化 学研究带来更为广阔的化学及专利空间. 如将环丙沙星 结构喹诺酮环 7 位哌嗪环替换为 2,6-二氮杂螺[3.3]环庚 烷后, 药物的抗菌活性不变, 而体内清除代谢率有显著 下降, 作用时间延长. 最近, 我们对部分四元杂环及四 元螺杂环类似物的合成进行研究, 并将其成功用于小分 子抗肿瘤激酶抑制剂的结构改造中 ${ }^{[4]}$. 鉴于 2,6-二氮杂 螺[3,4]环辛烷(图 1a)和 2-氧-6-氮杂螺[3,4]环辛烷(图 1b) 与哌嗪和吗啉在结构上的相似性, 同样在药物化学领域 具有良好的应用前景 ${ }^{[5]}$, 如采用 2,6 -二氮杂螺 $[3,4]$ 环辛 烷替换环丙沙星中的哌嗪, 得到了已进入临床三期研究 的化合物 zabofloxacin ${ }^{[6]}$.

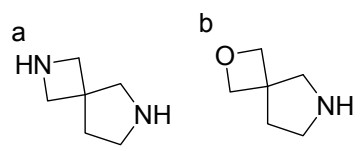

图 12 2,6-二氮杂螺[3,4]环辛烷和 2-氧-6-氮杂螺[3,4]环辛烷 Figure 1 2,6-Diazaspiro[3.4] octane and 2-oxa-6-azaspiro[3.4] octane

目前，关于 2,6 -二氮杂螺 $[3,4]$ 环辛烷的合成报道仅 有 Engel 等采用 $1,1,2$-乙烷三羧酸乙酯和 1,3,5-三芳基六 氢-1,3,5-三嗪为原料的方法 ${ }^{[7]}$. 而对于 2-氧-6-氮杂螺 $[3,4]$ 环辛烷的合成, Carreira ${ }^{[8]}$ 和 $\mathrm{Xu}$ 等 ${ }^{[9]}$ 的方法涉及了一 些比较严苛的实验条件 $\left(\right.$ 如 $\left.-78{ }^{\circ} \mathrm{C}\right)$ 、较贵的起始原料 (如氧杂环丁酮)以及剧毒试剂(如氯甲酸甲酯). 鉴于现 有合成方法中存在的不足，我们希望通过更简单、经济、 高效的方法完成 2,6-二氮杂螺 [3,4]环辛烷和 2-氧-6-氮杂 螺 $[3,4]$ 环辛烷的合成.

结合已有的五元环和四元环的合成方法 ${ }^{[10]}$, 我们

* E-mail: xcdong@fudan.edu.cn, zhaoweili@fudan.edu.cn

Received July 1, 2014; revised September 19, 2014; published online September 25, 2014.

Project supported by the Specialized Research Fund for the Doctoral Program of Higher Education of China (No. 20130071110071), the Shanghai Municipal Natural Science Foundation (No. 12ZR1403300), the Shanghai Municipal Science \& Technology Pillar Program for Bio-pharmaceuticals (No. 13431900102). 教育部博士点基金(No. 20130071110071)、上海市自然科学基金(No. 12ZR1403300)和上海市科技支撑(No. 13431900102)资助项目. 
采用先构建四氢吡咯环, 然后合成稳定性较差的四元杂 环的策略. 目前合成四氢吡咯环的主要方法有 3 种: 吡 咯环的氢化还原 ${ }^{[11]} 、 \delta$-卤代胺的分子内 $N$-烃化环合 ${ }^{[12]}$

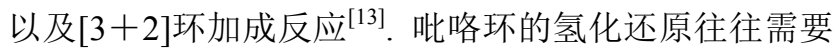
特殊的加压氢化设备, 而分子内 $N$-烃化环合能适用的 底物范围非常有限. 相比而言, [3+2]环加成反应的反 应条件温和, 底物适用性也更广, 因此我们选用这一方 法构建四氢吡咯环.

我们设计了两条路线(图 2, 路线 A, B)来构建四氢 吡咯环, 即以丙二酸二乙酯或丙烯酸乙酯为起始原料, 分别经 Knoevenagel 缩合或 Baylis-Hillman 反应得到中 间体 4 和 9, 化合物 4 或 9 与 $N$-甲氧甲基- $N$-三甲基硅甲 基茮胺经 $[3+2]$ 环加成反应得到四氢吡咯衍生物 $\mathbf{5}$ 或 10. 四氢吡咯环构建好后, 环外的两个取代基经过还原、取 代转化成具备更强离去能力的双甲磺酸酯 $\mathbf{6}$, 进一步与 对甲苯环酰胺关环得到氮杂环丁烷的化合物 7. 再经过 脱除保护基, 与草酸成盐, 目标化合物 6-茮基- 2,6-二氮 杂螺 $[3,4]$ 环辛烷草酸盐(1)即可制得. 采用同样的先构 建吡咯烷环的策略得到中间体 10 后, 再经过醇的甲磺 酸酯活化、还原、关环三步反应即可完成氧杂环丁烷的 构建(图 2, 路线 C). 最后, 脱除保护基即可合成目标化 合物 2-氧-6-氮杂螺 [3,4]环辛烷(2).

\section{1 结果与讨论}

\section{$1.12,6-$ 二氮杂螺 $[3,4]$ 环辛烷的合成}

\section{1 .1 合成路线 $\mathrm{A}$}

路线 $\mathrm{A}$ 的合成以丙二酸二乙酯 $(3)$ 为起始原料, 在 醋酸铜的催化下, 与多聚甲醛经 Knoevenagel 缩合反应 可生成甲撑丙二酸二乙酯(4) (Scheme 1). 这个反应遇到 的主要问题在于甲撑丙二酸二乙酯(4)极不稳定、后处理
过程也很繁琐 ${ }^{[14]}$. 该化合物与被广泛使用的黏合剂氰 基丙酸乙酯(商品名: 502 胶水)类似，因此也表现出黏合 剂的性质. 它自身可快速聚合, 在 $30 \mathrm{~min}$ 内而变成粘稠 的胶状物. 为了减少这种自身聚合的倾向, 往往需要向 化合物中添加稳定剂. 早期常被使用的经典稳定剂包括 顺丁烯二酸和对苯二酚 ${ }^{[15]}$. 然而, 即便添加 $0.1 \%$ 的顺丁 烯二酸和对苯二酚, 仍有约 $40 \%$ 的产物会发生自身聚 合. 为了提高反应的收率，通过耖选后我们发现 4-差基 TEMPO 对于抑制化合物 4 自身聚合的效果较好，它常 被作为稳定剂用于防止丙烯酸酯、丙烯酸、丙烯腈、苯 乙烯以及丁二烯类化合物的聚合.

我们在反应液中加入了 $0.1 \%$ 的 4-羟基 TEMPO (4羟基-2,2,6,6-四甲基哌啶 1-氧基自由基), 在蒸馏纯化前 加入了 $0.2 \%$ 的 4 -差基 TEMPO. 同时, 在蒸馏装置中的 收集瓶中, 我们也加入了 $0.1 \%$ 的 4-羟基 TEMPO. 经过 这样的优化，可有效抑制化合物 $\mathbf{4}$ 的自身聚合，其收率 可以达到 $75 \%$. 在下一步的 $[3+2]$ 环加成反应中，化合 物 4 和 $N$-甲氧甲基- $N$-(三甲基硅甲基)芐胺在三氟醋酸 的催化下能够以 $74 \%$ 的收率得到化合物 5 . 需要指出的 是, 如果不在化合物 $\mathbf{4}$ 中加入稳定剂, 化合物 $\mathbf{5}$ 的收率 将会低于 40\%. 经氢化锂铝的还原，化合物 5 的双酯基 被还原为双羟基，然后在 $-18{ }^{\circ} \mathrm{C}$ 的条件下，甲基磺酰 氯将双羟基转化为双甲磺酰基的化合物 6 . 这两步的合 并收率可达 $85 \%$.

\section{1 .2 合成路线 B}

鉴于化合物 4 双键两端的双吸电子基团取代所造成 的不稳定性, 我们尝试改用更稳定的中间体来进行 [3+ 2]环加成反应，所以我们设计了双键上只连接一个吸电 子基的中间体 9 . 如在路线 $\mathrm{B}$ 中, 丙烯酸乙酯 $(\mathbf{8})$ 先经 1,4-二氮杂二环[2.2.2]辛烷(DABCO) (0.1 equiv.)催化与

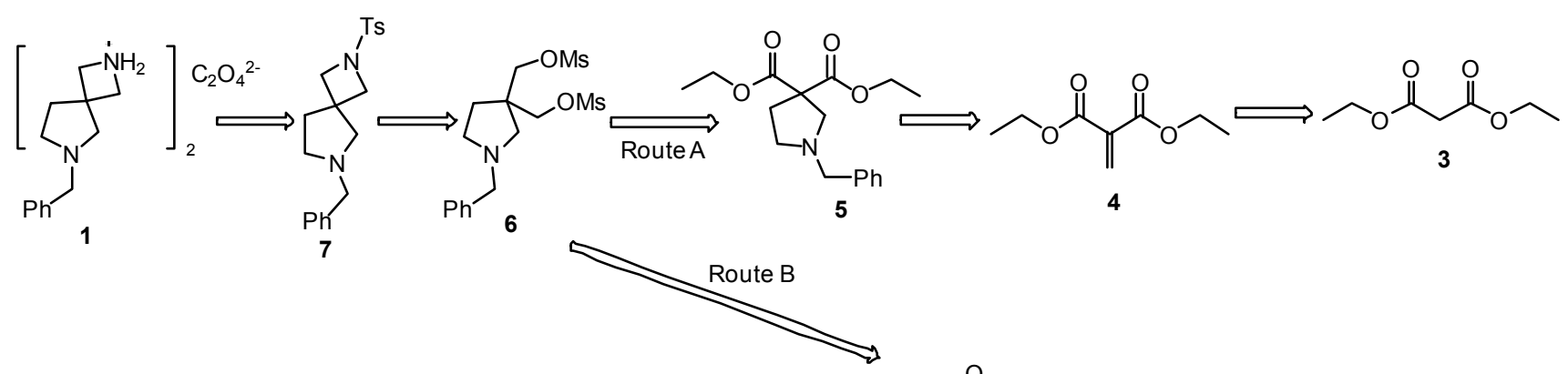

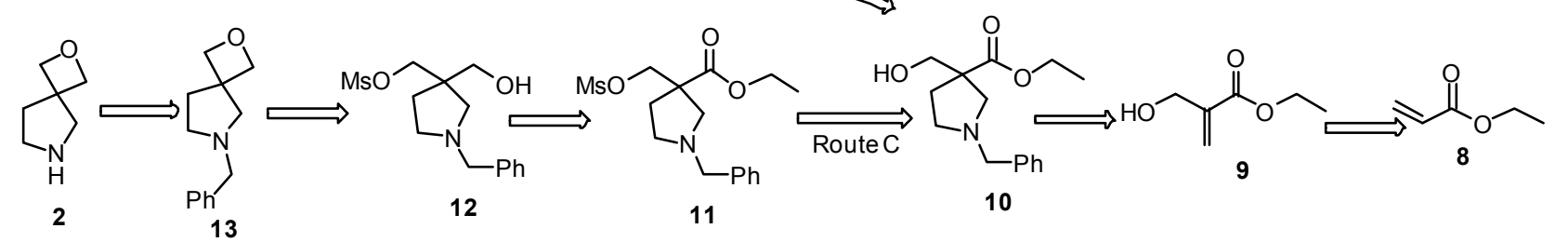

图 2 6-苠基-2,6-二氮杂螺[3,4]环辛烷和 2-氧-6-氮杂螺[3,4]环辛烷的逆合成分析

Figure 2 Synthetic routes of 6-benzyl-2,6-diazaspiro[3.4]octane oxalate and 2-oxa-6-azaspiro[3,4]octane 


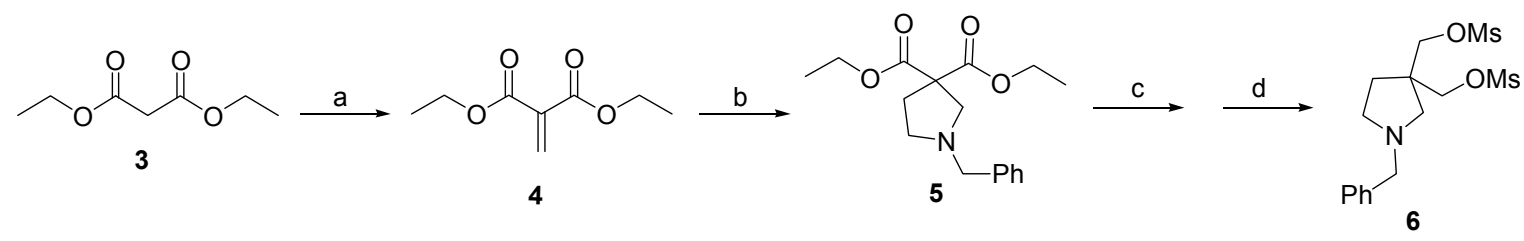

Reagents and conditions: (a) $\mathrm{Cu}(\mathrm{OAc})_{2} \cdot \mathrm{H}_{2} \mathrm{O}$ (0.32 equiv.), $\left(\mathrm{CH}_{2} \mathrm{O}\right)_{n}\left(2.6\right.$ equiv.), $\mathrm{HOAc}\left(2.4\right.$ equiv.), toluene, $90{ }^{\circ} \mathrm{C}, 90 \%$; (b) $\mathrm{N}$-benzyl-1-methoxy- $\mathrm{N}$-((trimethylsilyl)methyl)-methanamine (1.4 equiv.), $\mathrm{CF}_{3} \mathrm{COOH}(0.12$ equiv.)

图式 1 6-苠基-2,6-二氮杂螺[3,4]环辛烷(1)的合成路线 A

Scheme 1 Synthetic route A of 6-benzyl-2,6-diazaspiro[3.4] octane oxalate (1)

多聚甲醛通过 Baylis-Hillman 反应生成化合物 9 . 但在该 条件下, 产率仅有 $47 \%$ (Scheme 2) ${ }^{[16]}$. Hu 等 ${ }^{[17]}$ 报道将 $\mathrm{DABCO}$ 的等物质的量的比提高之后，化合物 9 的收率 可得到改善. 室温下, 将 1 equiv. 的醛和 3 equiv. 的丙烯 酸乙酯(8)溶于 1,4 -二氧六环和水(体积比 $1: 1$ )中, 再加 入 1 equiv.的 DABCO, 这样就可将收率提升到 $93 \%$. 但 在我们的反应中, 过量的丙烯酸乙酯会干扰后续的反 应. 因此在优化这个反应时, 我们首先把 DABCO 的比 例提升到 1 equiv., 但同时将多聚甲醛的比例也提高到 3 equiv., 以消耗掉所有的丙烯酸乙酯. 通过这样的改善, 我们以 $91 \%$ 的收率得到了化合物 $\mathbf{9}$. 化合物 9 在后处理、 纯化以及后续反应的过程中均不用加入稳定剂, 且都能 够获得较好的收率. 化合物 9 可采用与路线 $\mathrm{A}$ 中相同的 $[3+2]$ 环加成条件得到化合物 10. 化合物 10 的酯基经嗍 氢化钠还原为羟基, 然后采用与路线 $\mathrm{A}$ 相同的条件以较 高的收率得到双甲磺酰基的化合物 6 .

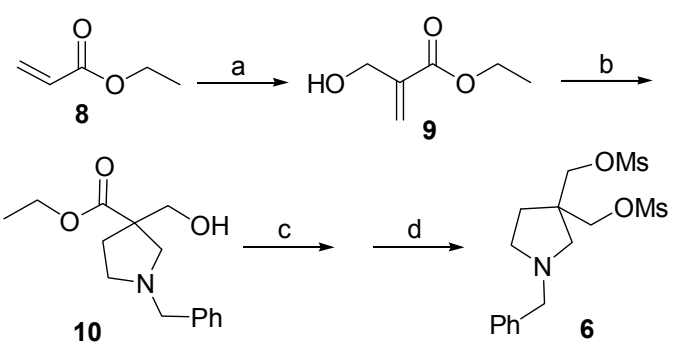

Reagents and conditions: (a) $\left(\mathrm{CH}_{2} \mathrm{O}\right)_{n}$ (3 equiv.), DABCO (1 equiv.), dioxane, $\mathrm{H}_{2} \mathrm{O}$, r.t., $91 \%$; (b) $\mathrm{N}$-benzyl-1-methoxy- $\mathrm{N}$-((trimethylsilyl)methyl) methanamine (1.1 equiv.), $\mathrm{CF}_{3} \mathrm{COOH}\left(0.1\right.$ equiv.), $0{ }^{\circ} \mathrm{C} \sim$ r.t., $71 \%$; (c) $\mathrm{NaBH}_{4}$ (3 equiv.), $\mathrm{EtOH}, 0{ }^{\circ} \mathrm{C} \sim$ r.t.; (d) $\mathrm{MsCl}$ (3 equiv.), $\mathrm{Et}_{3} \mathrm{~N}$ (3 equiv.), DCM, $-18{ }^{\circ} \mathrm{C}, 85 \%$ (for two steps).

图式 2 6-芐基-2,6-二氮杂螺 [3,4]环辛烷(1)的合成路线 B Scheme 2 Synthetic route B of 6-benzyl-2,6-diazaspiro[3.4]octane oxalate (1)

\subsection{3 氮杂环丁烷的构建}

在将化合物 6 环合为氮杂环丁烷的步骤中(Scheme 3 ), 我们筛选尝试了多种胺(表 1). 结果, 只有对甲苯磺 酰胺能够促成氮杂环丁烷的生成, 得到 6-芐基-2-对甲 苯磺酰基-2,6-二氮杂螺 [3,4]环辛烷(7). 对甲苯磺酰胺在 促成四元环环合中体现的有效性与之前的文献[18]报道
一致. 最后, 脱去对甲苯磺酰保护基，与草酸成盐得到 目标产物 $\mathbf{1}^{[3]}$.
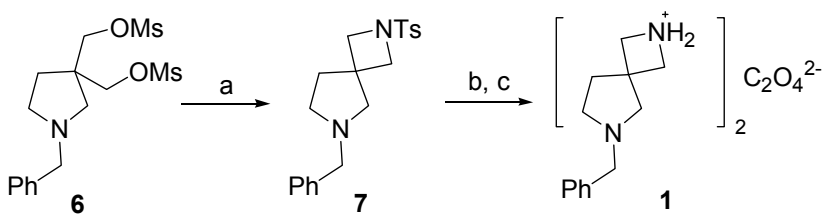

Reagents and conditions: (a) $\mathrm{TsNH}_{2}$ (1.6 equiv.), DMSO, $\mathrm{K}_{2} \mathrm{CO}_{3}$ (2.2 equiv.), $100{ }^{\circ} \mathrm{C}, 83 \%$; (b) $\mathrm{Mg}$ powder (6 equiv.), $\mathrm{MeOH}$, super sonicated, r.t.; (c) $\mathrm{H}_{2} \mathrm{C}_{2} \mathrm{O}_{4}$ (1 equiv.), $\mathrm{Et}_{2} \mathrm{O}$, $\mathrm{EtOH}$, r.t., $79 \%$ (for two steps).

图式 3 6-苠基-2,6-二氮杂螺 $[3,4]$ 环辛烷中氮杂四元环的环合 Scheme 3 Cyclization of azaetidine in 6-benzyl-2,6-diazaspiro[3.4]octane

表 1 四元环环合时胺的笁选

Table 1 The cyclization of disulfonate with amines

\begin{tabular}{llc}
\hline Amine & \multicolumn{1}{c}{ Condition } & Products of 2 \\
\hline $\mathrm{NH}_{3} \cdot \mathrm{H}_{2} \mathrm{O}$ & Sealed Tube $/ \mathrm{Et}_{3} \mathrm{~N} / \mathrm{H}_{2} \mathrm{O} / 100{ }^{\circ} \mathrm{C}$ & - \\
$\mathrm{CH}_{3} \mathrm{NH}_{2}$ & Sealed Tube $/ \mathrm{Et}_{3} \mathrm{~N} / \mathrm{EtOH} / 100{ }^{\circ} \mathrm{C}$ & - \\
$\mathrm{C}_{2} \mathrm{H}_{5} \mathrm{NH}_{2}$ & Sealed Tube $/ \mathrm{Et}_{3} \mathrm{~N} / \mathrm{EtOH} / 100{ }^{\circ} \mathrm{C}$ & - \\
$\mathrm{BnNH}_{2}$ & $\mathrm{DMSO} / \mathrm{K}_{2} \mathrm{CO}_{3} / 100{ }^{\circ} \mathrm{C}$ & - \\
$\mathrm{TosNH}_{2}$ & $\mathrm{DMSO} / \mathrm{K}_{2} \mathrm{CO}_{3} / 100{ }^{\circ} \mathrm{C}$ & + \\
$\mathrm{CbzNH}_{2}$ & $\mathrm{DMSO} / \mathrm{K}_{2} \mathrm{CO}_{3} / 100{ }^{\circ} \mathrm{C}$ & - \\
\hline
\end{tabular}

\subsection{2-氧-6-氮杂螺[3,4]环辛烷的合成}

2-氧-6-氮杂螺 $[3,4]$ 环辛烷通过路线 C 合成而得 (Scheme 4). 首先, 路线 B 中合成的中间体 $\mathbf{1 0}$ 与甲基磺 酰氯(1.5 equiv.)反应, 羊基被甲磺酰化, 得到化合物 $\mathbf{1 1}$. 化合物 11 的酯基经氢化锂铝(1.2 equiv.)还原为羟乙基, 得到化合物 12. 在这步还原反应中, 不能使用硼氢化 钠. 因为在硼氢化钠的碱性的影响下，甲氧基和甲磺酰 基可能发生交换生成副产物. 化合物 12 在 $\mathrm{NaH}$ (2.0 equiv.)的作用下, 可得到环合产物 $\mathbf{1 3}$, 三步反应的合并 收率可到 $71 \%$. 最后, 经过钯碳脱茮, 以 $80 \%$ 的收率得 到目标化合物 $\mathbf{2}^{[9]}$. 需要特别指出的是采用中间体 $\mathbf{1 0}$ 来合成 2-氧-6-氮杂螺 $[3,4]$ 环辛烷较 $\mathrm{Xu}$ 等 ${ }^{[9]}$ 的方法具有 选择性更好的优势. 

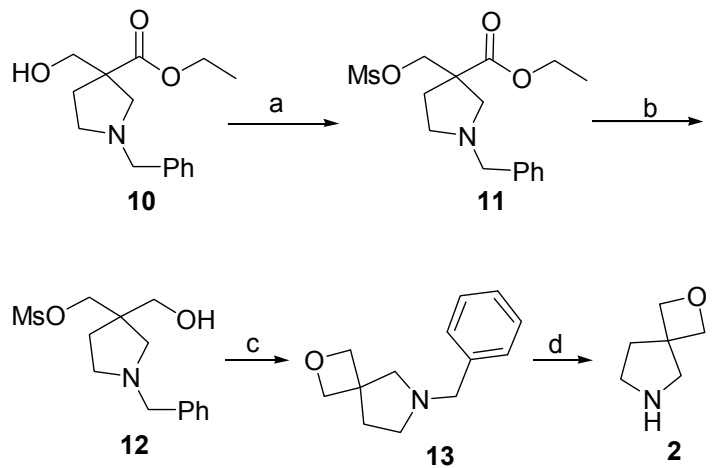

Reagents and conditions: (a) $\mathrm{Et}_{3} \mathrm{~N}$ (2 equiv.), $\mathrm{MsCl}$ (1.5 equiv.), DCM, $-18{ }^{\circ} \mathrm{C} \sim$ r.t.; (b) $\mathrm{LiAlH}_{4}$ (1.2 equiv.), THF, $0{ }^{\circ} \mathrm{C} \sim$ reflux; (c) $\mathrm{NaH}$ (2.0 equiv.), THF, reflux, $71 \%$ (for three steps); (d) $\mathrm{H}_{2}, \mathrm{Pd} / \mathrm{C}$, $\mathrm{MeOH}, 80 \%$

图式 4 2-氧-6-氮杂螺 $[3,4]$ 环辛烷(2)的合成路线 C Scheme 4 Synthetic route C of 2-oxa-6-azaspiro[3,4]octane (2)

\section{2 结论}

我们改进了 6-苄基-2,6-二氮杂螺 [3,4]环辛烷和 2氧-6-氮杂螺 [3,4]环辛烷的合成方法. 设计的 3 条合成路 线能够以克级水平快速制备两个四元螺环化合物, 起始 原料易得, 这也为它们进一步在药物化学中的应用奠定 了基础.

\section{3 实验部分}

\section{1 仪器与试剂}

所用试剂如无特殊说明均为市售分析纯或化学纯, 采购自 SCRC, Adamas, J\&K,Alfa Aesar, Acros, Aldrich 或 TCI, 并直接使用. 薄层层析硅胶用青岛海洋化工生 产 $\mathrm{HSGF}_{254}$ 高效板, 柱层析用硅胶 H (200 300 目). 核 磁共振氢谱 $\left({ }^{1} \mathrm{H}\right.$ NMR) 和碳谱 $\left({ }^{13} \mathrm{C}\right.$ NMR) 采用 Bruker Avance $400 \mathrm{MHz}$ 或 $600 \mathrm{MHz}$ 核磁共振仪测定; 质谱 (ESI-MS)采用 Aglient 6120 单四级杆质谱仪测定; 高分 辨质谱(HRMS)采用 $\mathrm{AB} 5600+\mathrm{Q}$ TOF 高分辨质谱仪测 定.

\section{2 实验方法}

\subsection{1 甲撑丙二酸二乙酯(4)的合成}

室温下, 依次将多聚甲醛 $(21.70 \mathrm{~g}, 0.72 \mathrm{~mol})$ 、醋酸 铜(18.83 g, $0.09 \mathrm{~mol}) 、$ 冰醋酸 $(39.54 \mathrm{~g}, 0.66 \mathrm{~mol})$ 和甲苯 $(200 \mathrm{~mL})$ 加入 Dean-Stark 蒸馏器中. 升温至 $90{ }^{\circ} \mathrm{C}$, 搅拌 $3 \mathrm{~h}$. 然后用恒压低液漏斗滴加丙二酸二乙酯 $(45.00 \mathrm{~g}$, $0.28 \mathrm{~mol})$. 滴毕, 反应回流 $3 \mathrm{~h}$, Dean-Stark 蒸馏器中收 集水 $(6 \mathrm{~mL})$. 冷却至室温, 过滤除盐. 向滤液中加入 4羟基 TEMPO $(0.30 \mathrm{~g}, 0.5 \% \mathrm{~W} / \mathrm{W})$. 滤液减压浓缩去除甲 苯, 得到棕色液体粗品 $(65.23 \mathrm{~g})$. 粗品经减压蒸馏 $\left(80{ }^{\circ} \mathrm{C} / 10 \mathrm{mmHg}\right.$ ) 得 $43.39 \mathrm{~g}$ 黄色液体 $4^{[19]}$, 产率 $90 \%$.
最后，需向纯品中加入 4-羟基 TEMPO (0.18 g, 0.5\% $W / W$ ) 以稳定产物. 薄层层析 (TLC): $R_{\mathrm{f}}=0.60[V($ 石油 醚) $: V($ 乙酸乙酯 $\left.)=2: 1 ; \mathrm{UV}, \mathrm{I}_{2}\right]$.

\subsubsection{1-苄基吡咯烷基-3,3-二甲酸乙酯(5)的合环}

冰浴下, 将 $N$-甲氧甲基- $N$-(三甲基硅甲基)芐胺 $(33.60 \mathrm{~g}, 0.14 \mathrm{~mol})$ 和甲撑丙二酸二乙酯(4) (17.20 g, 0.10 $\mathrm{mol})$ 溶于二氯甲烷 $(200 \mathrm{~mL})$, 三氟乙酸 $(1.32 \mathrm{~g}, 0.012$ $\mathrm{mol}$ )缓慢滴加入反应体系中, 滴加完后冰浴下搅拌 $1 \mathrm{~h}$, 再升温至 $25{ }^{\circ} \mathrm{C}$ 下继续搅拌 $12 \mathrm{~h}$ 后, 倒入饱和碳酸氢钠 溶液 $(100 \mathrm{~mL})$, 搅拌 $10 \mathrm{~min}$, 分液. 有机层用饱和碳酸 氢钠溶液和饱和氯化钠溶液各洗 1 次, 无水硫酸钠干燥. 过滤, 硅胶柱层析纯化 $[V$ (石油醚) $: V$ (乙酸乙酯 $)=20$ : 1]得到 $26.85 \mathrm{~g}$ 淡黄色液体 $5^{[20]}$, 产率 74\%. ${ }^{1} \mathrm{H}$ NMR (400 $\left.\mathrm{MHz}, \mathrm{CDCl}_{3}\right) \delta: 7.36 \sim 7.29(\mathrm{~m}, 4 \mathrm{H}), 7.27 \sim 7.25(\mathrm{~m}, 1 \mathrm{H})$, $4.24 \sim 4.18(\mathrm{~m}, 4 \mathrm{H}), 3.65(\mathrm{~s}, 2 \mathrm{H}), 3.07(\mathrm{~s}, 2 \mathrm{H}), 2.69(\mathrm{t}, J=$ $6.9 \mathrm{~Hz}, 2 \mathrm{H}), 2.46(\mathrm{t}, J=6.9 \mathrm{~Hz}, 2 \mathrm{H}), 1.26(\mathrm{t}, J=7.1 \mathrm{~Hz}$, $6 \mathrm{H}) ;{ }^{13} \mathrm{C}$ NMR $\left(100 \mathrm{MHz}, \mathrm{CDCl}_{3}\right) \delta: 171.3,138.7,128.5$, $128.2,127.0,61.5,60.0,59.4,59.3,53.4,32.6,14.0 ; \mathrm{MS}$ (ESI) $m / z: 306.1[\mathrm{M}+\mathrm{H}]^{+}$.

3.2 .31 - 苄基-3,3-二[(甲磺酰基氧基)甲基]-吡咯 $(\mathbf{6})$ 的合成

方法 1 (从 5 合成 6 , 路线 A): 在 $0{ }^{\circ} \mathrm{C}$ 、氩气保护下, 向干燥的装有氢化锂铝 $(5.00 \mathrm{~g}, 0.13 \mathrm{~mol})$ 的三颈瓶中, 加入无水四氢呋喃 $(100 \mathrm{~mL})$, 滴加溶有化合物 5 (10.00 $\mathrm{g}, 0.033 \mathrm{~mol})$ 的无水四氢呋喃 $(50 \mathrm{~mL})$ 溶液, 约 $1 \mathrm{~h}$ 滴完 后, 升温至 $25{ }^{\circ} \mathrm{C}$ 搅拌 $2 \mathrm{~h}$. 然后, 将反应缓慢倒入至饱 和氯化铵溶液中, 经硅藻土过滤. 滤液分层, 水层用乙 酸乙酯 $(100 \mathrm{~mL})$ 萃取 5 次. 合并有机层, 减压蒸干. 将残 余物溶于二氯甲烷 $(200 \mathrm{~mL})$ 中, 用饱和食盐水洗涤后, 无水硫酸钠干燥. 过滤, 在 $-18{ }^{\circ} \mathrm{C}$ 下, 依次加入三乙胺 $(10.10 \mathrm{~g}, 0.10 \mathrm{~mol})$ 和甲磺酰氯 $(11.3 \mathrm{~g}, 0.10 \mathrm{~mol})$, 并在该 温度下反应 $2 \mathrm{~h}$. 倒入饱和碳酸钠溶液, 搅拌 $10 \mathrm{~min}$, 静 置, 分层. 水层用二氯甲烷 $(100 \mathrm{~mL})$ 萃取 3 遍. 合并有机 层, 无水硫酸钠干燥, 过滤, 得到 $10.59 \mathrm{~g}$ 无色液体 $\mathbf{6}$, 产率 $85 \%$. 该粗品可不经纯化直接用于下一步反应.

方法 2(从 10 合成 6, 路线 B)：将化合物 $\mathbf{1 0}(18.5 \mathrm{~g}$, $0.07 \mathrm{~mol}$ ) 溶于甲醇 $\left(250 \mathrm{~mL}\right.$ ), 冷却至 $0{ }^{\circ} \mathrm{C}$, 分批加入嗍 氢化钠 $(8.0 \mathrm{~g}, 0.21 \mathrm{~mol})$. 加完后移去冰浴, 回流 $5 \mathrm{~h}$. 冷 却至室温, 将反应液倒入饱和氯化铵溶液 $(200 \mathrm{~mL})$ 中, 水相用乙酸乙酯 $(100 \mathrm{~mL})$ 萃取 5 次, 合并有机层, 减压 蒸干, 用二氯甲烷溶解浓缩所得的粗品, 饱和氯化钠溶 液洗后无水硫酸钠干燥, 过滤, 滤液直接加入三乙胺 (21.25 g, $0.21 \mathrm{~mol})$, 冷却至 $-18{ }^{\circ} \mathrm{C}$, 滴加甲基磺酰氯 $(24.06 \mathrm{~g}, 0.21 \mathrm{~mol})$, 并在该温度下搅拌 $12 \mathrm{~h}$. 倒入饱和 碳酸钠溶液, 搅拌 $10 \mathrm{~min}$, 分液. 水相用二氯甲烷(100 
$\mathrm{mL}$ )萃取三次, 合并有机层, 用无水硫酸钠干燥, 过滤, 滤液减压浓缩得到 $22.46 \mathrm{~g}$ 无色液体粗品 6 , 粗品产率 $85 \%$, 不经纯化直接投入下一步反应. ${ }^{1} \mathrm{H}$ NMR (400 $\left.\mathrm{MHz}, \mathrm{CDCl}_{3}\right) \delta: 7.34 \sim 7.24(\mathrm{~m}, 5 \mathrm{H}), 4.21 \sim 4.16(\mathrm{~m}, 4 \mathrm{H})$, 3.59 (s, 2H), 3.01 (s, 6H), 2.64 (t, $J=6.9 \mathrm{~Hz}, 2 \mathrm{H}), 2.44$ (s, $2 \mathrm{H}), 1.72(\mathrm{t}, J=6.9 \mathrm{~Hz}, 2 \mathrm{H}) ;{ }^{13} \mathrm{C} \mathrm{NMR}\left(100 \mathrm{MHz}, \mathrm{CDCl}_{3}\right)$ $\delta: 126.1,125.8,124.7,68.4,56.9,56.2,50.4,43.0,34.7$, 27.9, 27.2; HRMS calcd for $\mathrm{C}_{16} \mathrm{H}_{24} \mathrm{NO}_{6} \mathrm{~S}_{2}[\mathrm{M}+\mathrm{H}]^{+}$ 378.1040 , found 378.1043 .

3.2.4 6-芳基-2-对甲苯磺酰基-2,6-二氮杂 $[3,4]$ 环辛 烷(7)的合成

将化合物 6 (18.87 g, $0.05 \mathrm{~mol}$ )溶于二甲基亚砜(50 $\mathrm{mL})$ 中, 再依次加入碳酸钾 $(10.50 \mathrm{~g}, 0.11 \mathrm{~mol})$ 和对甲苯 磺酰胺 $(13.60 \mathrm{~g}, 0.08 \mathrm{~mol})$. 加热至 $100{ }^{\circ} \mathrm{C}$ 搅拌 $24 \mathrm{~h}$, 冷 却到室温. 将反应液倒入水 $(200 \mathrm{~mL})$ 和乙酸乙酯 $(200$ $\mathrm{mL})$ 中, 分液, 水层用乙酸乙酯 $(100 \mathrm{~mL})$ 萃取 3 次. 合并 有机层, 用水 $(100 \mathrm{~mL})$ 洗 3 次, 然后用无水硫酸钠干燥. 过滤, 滤液减压浓缩, 得到红褐色液体粗品. 粗品经硅 胶柱层析纯化 $[V$ (二氯甲烷 $): V$ (甲醇) $: V$ (氨水 $)=500$ : $10: 2$ ]得到 $15.75 \mathrm{~g}$ 无色液体 $7^{[21]}$, 产率 $83 \% .{ }^{1} \mathrm{H}$ NMR $\left(400 \mathrm{MHz}, \mathrm{CDCl}_{3}\right) \delta: 7.70(\mathrm{~d}, J=8.1 \mathrm{~Hz}, 2 \mathrm{H}), 7.35 \sim 7.23$ $(\mathrm{m}, 7 \mathrm{H}), 3.69 \sim 3.64(\mathrm{~m}, 4 \mathrm{H}), 3.52(\mathrm{~s}, 2 \mathrm{H}), 2.51 \sim 2.43(\mathrm{~m}$, $7 \mathrm{H}), 1.83(\mathrm{t}, J=7.1 \mathrm{~Hz}, 2 \mathrm{H}) ;{ }^{13} \mathrm{C}$ NMR $\left(100 \mathrm{MHz}, \mathrm{CDCl}_{3}\right)$ $\delta: 143.9,138.3,131.1,129.6,128.5,128.2,128.2,127.0$, $64.4,62.3,59.8,53.1,38.9,36.5,21.5$; MS (ESI) $m / z$ : $357.2[\mathrm{M}+\mathrm{H}]^{+}$.

\subsubsection{2-羟甲基丙烯酸乙酯(9)的合成}

室温下, 依次将多聚甲醛 $(20.0 \mathrm{~g}, 0.66 \mathrm{~mol}) 、$ 丙烯酸 乙酯 $(22.0 \mathrm{~g}, 0.22 \mathrm{~mol})$ 和 DABCO $(24.6 \mathrm{~g}, 0.22 \mathrm{~mol})$ 溶于 1,4 -二氧六环/水 $(200 \mathrm{~mL}, V: V=1: 1)$, 搅拌 $4 \mathrm{~h}$. 然后, 减压蒸去 1,4-二氧六环, 加入乙醚和水各 $200 \mathrm{~mL}$, 分 液, 水层乙醚 $(150 \mathrm{~mL})$ 萃取 3 次, 合并有机层, 饱和氯化 钠 $(100 \mathrm{~mL})$ 洗. 无水硫酸镁干燥, 过滤, 减压蒸干, 硅胶 柱层析纯化 $[V$ (石油醚)： $V$ (乙酸乙酯) $=1: 1$ ] 得到 26.00 $\mathrm{g}$ 无色油状液体 $\mathbf{9}^{[22]}$, 产率 $91 \% .{ }^{1} \mathrm{H}$ NMR $(400 \mathrm{MHz}$, $\left.\mathrm{CDCl}_{3}\right) \delta: 6.20(\mathrm{~d}, J=1.0 \mathrm{~Hz}, 1 \mathrm{H}), 5.79(\mathrm{~d}, J=1.4 \mathrm{~Hz}$, $1 \mathrm{H}), 4.27 \sim 4.25(\mathrm{~m}, 2 \mathrm{H}), 4.20 \sim 4.15(\mathrm{~m}, 2 \mathrm{H}), 3.05(\mathrm{t}, J=$ $6.3 \mathrm{~Hz}, 1 \mathrm{H}), 1.26$ (t, $J=7.1 \mathrm{~Hz}, 3 \mathrm{H}$ ); MS (ESI) $m / z: 131.1$ $[\mathrm{M}+\mathrm{H}]^{+}$.

\subsubsection{1-芐基-3-(羟甲基)吡咯烷基-3-羧酸乙酯(10)的} 合成

冰浴下, 将 $N$-甲氧甲基- $N$-(三甲基硅甲基)芐胺 (32.0 g, $0.13 \mathrm{~mol})$ 和化合物 9 (16.0 g, $0.12 \mathrm{~mol})$ 溶于二氯 甲烷 $(200 \mathrm{~mL})$, 再将三氟乙酸 $(1.32 \mathrm{~g}, 0.012 \mathrm{~mol}$ )缓慢滴 加至反应体系中, 滴加完后冰浴下搅拌 $1 \mathrm{~h}$, 撤去冰浴
于 $25{ }^{\circ} \mathrm{C}$ 下继续搅拌 $12 \mathrm{~h}$. 倒入饱和碳酸氢钠溶液, 搅 拌 $10 \mathrm{~min}$, 分液. 有机层用饱和碳酸氢钠溶液和饱和氯 化钠溶液各洗 1 次, 无水硫酸钠干燥. 过滤, 减压蒸干, 硅胶柱层析纯化 $[V($ 石油醚 $) ： V($ 乙酸乙酯 $)=4: 1]$ 得到 $23.1 \mathrm{~g}$ 淡黄色液体 10, 产率 $71 \%$. ${ }^{1} \mathrm{H}$ NMR $(400 \mathrm{MHz}$, DMSO- $\left.d_{6}\right) \delta: 7.30 \sim 7.19(\mathrm{~m}, 5 \mathrm{H}), 4.10 \sim 4.05(\mathrm{~m}, 2 \mathrm{H})$, $3.61 \sim 3.52(\mathrm{~m}, 4 \mathrm{H}), 2.81(\mathrm{~d}, J=9.6 \mathrm{~Hz}, 1 \mathrm{H}), 2.54 \sim 2.44$ $(\mathrm{m}, 3 \mathrm{H}), 2.24 \sim 2.11(\mathrm{~m}, 1 \mathrm{H}), 1.74 \sim 1.70(\mathrm{~m}, 1 \mathrm{H}), 1.17(\mathrm{t}$, $J=7.1 \mathrm{~Hz}, 3 \mathrm{H}) ;{ }^{13} \mathrm{C}$ NMR (100 MHz, DMSO- $\left.d_{6}\right) \delta: 175.3$, $139.5,128.7,128.5,127.1,67.3,60.5,59.7,59.5,55.2$, 53.6, 31.4, 14.4; HRMS for $\mathrm{C}_{15} \mathrm{H}_{22} \mathrm{NO}_{3}[\mathrm{M}+\mathrm{H}]^{+}$calcd 264.1594, found 264.1597.

3.2.7 1- 芐基-3-[[(甲磺酰基)氧基]甲基]吡咯烷基-3羧酸乙酯(11)的合成

在一 $18{ }^{\circ} \mathrm{C}$ 下, 将化合物 $\mathbf{1 0}(8.00 \mathrm{~g}, 0.03 \mathrm{~mol})$ 、三乙 胺 $(8.3 \mathrm{~mL}, 0.06 \mathrm{~mol})$ 依次加入二氯甲烷 $(250 \mathrm{~mL})$ 中, 滴 加甲基磺酰氯 $(5.2 \mathrm{~g}, 0.045 \mathrm{~mol})$, 滴加完保持温度摚拌 $12 \mathrm{~h}$. 倒入饱和碳酸钠溶液 $(100 \mathrm{~mL})$, 搅拌 $5 \mathrm{~min}$, 分液. 水层用二氯甲烷 $(50 \mathrm{~mL})$ 萃取 3 次. 合并有机层, 用饱和 氯化钠溶液 $(50 \mathrm{~mL}$ )洗 1 次, 无水硫酸钠干燥. 过滤, 减 压浓缩得到 $10.11 \mathrm{~g}$ 黄色液体 11, 粗品产率 $98 \%$, 可不 经纯化直接投入下一步反应. ${ }^{1} \mathrm{H}$ NMR $(400 \mathrm{MHz}$, $\left.\mathrm{CDCl}_{3}\right) \delta: 7.33 \sim 7.22(\mathrm{~m}, 5 \mathrm{H}), 4.40 \sim 4.33(\mathrm{~m}, 2 \mathrm{H})$, $4.22 \sim 4.16(\mathrm{~m}, 2 \mathrm{H}), 3.62(\mathrm{~s}, 2 \mathrm{H}), 2.96(\mathrm{~s}, 3 \mathrm{H}), 2.83(\mathrm{td}$, $J=8.1,3.9 \mathrm{~Hz}, 1 \mathrm{H}), 2.75(\mathrm{~s}, 2 \mathrm{H}), 2.55 \sim 2.48(\mathrm{~m}, 1 \mathrm{H})$, 2.27 (ddd, $J=13.3,8.0,3.9 \mathrm{~Hz}, 1 \mathrm{H}), 1.83$ (dt, $J=13.5,7.8$ $\mathrm{Hz}, 1 \mathrm{H}), 1.27$ (t, $J=7.1 \mathrm{~Hz}, 3 \mathrm{H}) ;{ }^{13} \mathrm{C}$ NMR $(100 \mathrm{MHz}$, DMSO- $\left.d_{6}\right) \delta: 173.3,138.3,128.5,128.3,127.1,72.8,61.5$, $59.3,58.8,53.2,52.1,37.0,31.6,14.1$; HRMS for $\mathrm{C}_{16} \mathrm{H}_{24} \mathrm{NO}_{5} \mathrm{~S}[\mathrm{M}+\mathrm{H}]^{+}$calcd 342.1370, found 342.1372.

3.2.8 [1-芐基-3-(羟甲基)吡咯烷-3-基]甲基甲磺酸酯 (12)的合成

冰浴下, 将化合物 11 (10.0 g, $0.03 \mathrm{~mol})$ 溶于无水四 氢呋喃 $(100 \mathrm{~mL})$, 将该溶液滴加至溶有氢化锂铝 $(1.40 \mathrm{~g}$, $0.036 \mathrm{~mol})$ 的无水四氢呋喃 $(50 \mathrm{~mL})$ 溶液中. 滴加完后撤 去冰浴让反应体系逐渐回复到室温, 摚拌 $2 \mathrm{~h}$. 把反应 液倒入冰水 $(200 \mathrm{~mL})$ 中, 搅拌 $30 \mathrm{~min}$, 倒入饱和氯化铵 溶液 $(100 \mathrm{~mL})$ 和乙酸乙酯 $(200 \mathrm{~mL})$. 再经硅藻土过滤, 分液. 水层用乙酸乙酯 $(100 \mathrm{~mL})$ 萃取 3 次. 合并有机层, 无水硫酸镁干燥, 过滤, 减压蒸干, 得 $7.10 \mathrm{~g}$ 无色液体 12, 粗品产率 $81 \%$, 可不经纯化直接用于下一步反应. ${ }^{1} \mathrm{H}$ NMR $\left(600 \mathrm{MHz}, \mathrm{CDCl}_{3}\right) \delta$ : $7.34 \sim 7.27(\mathrm{~m}, 5 \mathrm{H})$, $4.21 \sim 4.18(\mathrm{~m}, 2 \mathrm{H}), 3.63 \sim 3.57(\mathrm{~m}, 4 \mathrm{H}), 3.01(\mathrm{~s}, 3 \mathrm{H})$, $2.97(\mathrm{dd}, J=8.7,1.8 \mathrm{~Hz}, 1 \mathrm{H}), 2.70(\mathrm{td}, J=8.5,5.7 \mathrm{~Hz}$, $1 \mathrm{H}), 2.57$ (td, $J=8.7,6.4 \mathrm{~Hz}, 1 \mathrm{H}), 2.52(\mathrm{~d}, J=9.8 \mathrm{~Hz}$, 
1H), $2.41(\mathrm{~d}, J=9.8 \mathrm{~Hz}, 1 \mathrm{H}), 1.79 \sim 1.74(\mathrm{~m}, 1 \mathrm{H}), 1.68$ (ddd, $J=13.5,8.2,5.5 \mathrm{~Hz}, 1 \mathrm{H}) ;{ }^{13} \mathrm{C}$ NMR $(100 \mathrm{MHz}$, $\left.\mathrm{CDCl}_{3}\right) \delta: 138.5,128.7,128.4,127.2,72.3,66.2,59.9$, 59.7, 53.3, 46.9, 37.1, 30.3; HRMS for $\mathrm{C}_{14} \mathrm{H}_{22} \mathrm{NO}_{4} \mathrm{~S}$ $[\mathrm{M}+\mathrm{H}]^{+}$calcd 300.1264 , found 300.1262.

\section{2 .9 6-芐基-2-氧-6-氮杂螺 $[3,4]$ 环辛烷(13)的合成}

冰浴下, 将化合物 12 (7.1 g, $0.024 \mathrm{~mol})$ 溶于无水四 氢呋喃 $(20 \mathrm{~mL})$, 分批加入氢化钠 $(1.9 \mathrm{~g}, 60 \%, 0.048$ $\mathrm{mol}$ ). 加毕后撤去冰浴, 加热回流 $14 \mathrm{~h}$. 将反应液倒入 冰水中淬灭反应，乙酸乙酯 $(100 \mathrm{~mL})$ 萃取 3 次，合并有 机层无水硫酸钠干燥, 过滤, 减压旋除所有溶剂得到粗 品. 粗品经硅胶柱层析 $[V$ (二氯甲烷 $): V$ (甲醇 $)=40 ： 1]$ 得到 $4.30 \mathrm{~g}$ 黄色液体 $13^{[9]}$, 产率 $90 \%$. ${ }^{1} \mathrm{H}$ NMR (400 $\left.\mathrm{MHz}, \mathrm{CDCl}_{3}\right) \delta: 7.34 \sim 7.24(\mathrm{~m}, 5 \mathrm{H}), 4.62 \sim 4.58(\mathrm{~m}, 4 \mathrm{H})$, 3.60 (s, 2H), 2.82 (s, 2H), $2.55(\mathrm{t}, J=7.1 \mathrm{~Hz}, 2 \mathrm{H}), 2.13$ (t, $J=7.1 \mathrm{~Hz}, 2 \mathrm{H})$; MS (ESI) $m / z: 204.1[\mathrm{M}+\mathrm{H}]^{+}$.

\subsubsection{2-氧-6-氮杂螺 $[3,4]$ 环辛烷 (2)的合成}

室温下，依次将化合物 13 (0.50 g, $2.46 \mathrm{mmol}) 、 \mathrm{Pd}$ (5\%固载于碳， $0.125 \mathrm{~g}, 25 \% \mathrm{~m} / \mathrm{m}$ )加入甲醇 $(20 \mathrm{~mL})$ 中, 氩气保护下加热回流 $6 \mathrm{~h}$, 冷却至室温后过滤, 滤液减 压蒸去溶剂, 得到 $0.20 \mathrm{~g}$ 淡黄色液体 $2^{[9]}$, 产率 $80 \%$. ${ }^{1} \mathrm{H}$ NMR (400 MHz, $\left.\mathrm{CDCl}_{3}\right) \delta: 4.57(\mathrm{~d}, J=6.3 \mathrm{~Hz}, 3 \mathrm{H}), 4.46$ (d, $J=6.2 \mathrm{~Hz}, 2 \mathrm{H}), 3.35$ (s, 2H), 3.11 (t, $J=7.4 \mathrm{~Hz}, 2 \mathrm{H})$, $2.19(\mathrm{t}, J=7.4 \mathrm{~Hz}, 2 \mathrm{H}) ;{ }^{13} \mathrm{C}$ NMR (100 MHz, DMSO- $\left.d_{6}\right)$ $\delta: 79.8,52.3,45.1,44.0,34.6$; MS (ESI) $m / z: 114.0[\mathrm{M}+$ $\mathrm{H}]^{+}$.

3.2.116-苄基-2,6-二氮杂螺 $[3,4]$ 环辛烷草酸盐(1)的 合成

将化合物 7 (7.00 g, $0.02 \mathrm{~mol})$ 溶于甲醇 $(300 \mathrm{~mL})$, 再 加入镁粉(3.00 g, $0.12 \mathrm{~mol}$ ), 超声 $2 \mathrm{~h}$. 减压蒸去溶剂, 向残余物中加入乙醚 $(100 \mathrm{~mL})$ 和十水硫酸钠 $(6.20 \mathrm{~g}$, $0.02 \mathrm{~mol})$. 再将该悬浊液在室温下剧烈搅拌 $2 \mathrm{~h}$, 然后经 硅藻土过滤. 滤液用无水硫酸钠干燥, 过滤. 摚拌下向 得到的滤液中加入无水草酸 $(1.30 \mathrm{~g}, 0.02 \mathrm{mmol})$ 的无水 乙醇 $(4 \mathrm{~mL})$ 溶液, 生成大量白色沉淀. 过滤, 干燥后得 到 $4.60 \mathrm{~g}$ 白色固体 $\mathbf{1}^{[21]}$, 产率 79\%. ${ }^{1} \mathrm{H}$ NMR (400 MHz, DMSO- $\left.d_{6}\right) \delta: 7.35(\mathrm{~s}, 1 \mathrm{H}), 7.34 \sim 7.26(\mathrm{~m}, 5 \mathrm{H}), 3.91(\mathrm{~d}$, $J=10.6 \mathrm{~Hz}, 2 \mathrm{H}), 3.83$ (d, $J=10.7 \mathrm{~Hz}, 2 \mathrm{H}), 3.60$ (s, 2H), $2.73(\mathrm{~s}, 2 \mathrm{H}), 2.53 \sim 2.51(\mathrm{~m}, 2 \mathrm{H}), 2.05(\mathrm{t}, J=7.1 \mathrm{~Hz}, 2 \mathrm{H})$; ${ }^{13} \mathrm{C}$ NMR (100 MHz, DMSO- $\left.d_{6}\right) \delta: 165.2,138.9,129.0$, 128.7, 127.5, 64.0, 59.4, 56.7, 53.1, 42.5, 36.2; MS (ESI) $\mathrm{m} / \mathrm{z}: 203.1[\mathrm{M}+\mathrm{H}]^{+}$.

辅助材料(Supporting Information) 化合物的核磁共 振氢谱、核磁共振碳谱和高分辨质谱. 这些材料可以免 费从本刊网站(http://sioc-journal.cn/)上下载.

\section{References}

[1] Lameijer, E. W.; Kok, J. N.; Back, T.; Jzerman, A. P. J. Chem. Inf. Model. 2006, 46, 553.

[2] Law, V.; Knox, C.; Djoumbou, Y.; Jewison, T.; Guo, A. C.; Liu, Y.; Maciejewski, A.; Arndt, D.; Wilson, M.; Neveu, V.; Tang, A.; Gabriel, G.; Ly, C.; Adamjee, S.; Dame, Z. T.; Han, B.; Zhou, Y.; Wishart, D. S. Nucleic Acids Res. 2014, 42(D1), D1091.

[3] (a) Wuitschik, G.; Rogers-Evans, M.; Müller, K.; Fischer, H.; Wagner, B.; Schuler, F.; Polonchuk, L.; Carreira, E. M. Angew. Chem., Int. Ed. 2006, 45, 7736 .

(b) Burkhard, J.; Carreira, E. M. Org. Lett. 2008, 10, 3525.

(c) Wuitschik, G.; Carreira, E. M.; Wagner, B.; Fischer, H.; Parrilla, I.; Schuler, F.; Rogers-Evans, M.; Müller, K. J. Med. Chem. 2010, $53,3227$.

(d) Li, D. B.; Rogers-Evans, M.; Carreira, E. M. Org. Lett. 2013, 15,4766 .

(e) Burkhard, J. A.; Guérot, C., Knust, H.; Carreira, E. M. Org. Lett. 2012, 14, 66.

(f) Burkhard, J. A.; Guérot, C.; Knust, H.; Rogers-Evans, M.; Carreira, E. M. Org. Lett. 2010, 12, 1944.

(g) Li, D. B.; Rogers-Evans, M.; Carreira, E. M. Org. Lett. 2011, 13, 6134 .

(h) Guérot, C.; Tchitchanov, B. H.; Knust, H.; Carreira, E. M. Org. Lett. 2011, 13, 780 .

(i) Burkhard, J. A.; Wuitschik, G.; Rogers-Evans, M.; Müller, K.; Carreira, E. M. Angew. Chem., Int. Ed. 2010, 49, 9052.

(j) Wuitschik, G.; Rogers-Evans, M.; Buckl, A.; Bernasconi, M.; Märki, M.; Godel, T.; Fischer, H.; Wagner, B.; Parrilla, I.; Schuler, F.; Schneider, J.; Alker, A.; Schweizer, W. B.; Müller, K.; Carreira, E. M. Angew. Chem., Int. Ed. 2008, 47, 4512 .

(k) Burkhard, J. A.; Wagner, B.; Fischer, H.; Schuler, F.; Müller, K.; Carreira, E. M. Angew. Chem., Int. Ed. 2010, 49, 3524.

[4] (a) Zhao, Y. C.; Wang, W.; Li, F.; Wang, F.; Zheng, X. F.; Yun, H. Y.; Zhao, W. L.; Dong, X. C. Tetrahedron Lett. 2013, 54, 5849.

(b) Zhao, F.; Lin, Z. H.; Wang, F.; Zhao, W. L.; Dong, X. C. Bioorg. Med. Chem. Lett. 2013, 23, 5385.

(c) Zhao, W. L.; Dong, X. C.; Zhang, W. X.; Wang, F.; Li, J.; Zhao, Y. C.; Zhao, F.; Wang, W. CN 102796110, 2012 [Chem. Abstr. 2012, 158, 56273].

(d) Zhao, W. L.; Dong, X. C.; Zhang, W. X.; Wang, F.; Li, J.; Zhao, Y. C.; Zhao, F.; Wang, W. CN 102796109, 2012 [Chem. Abstr. 2012, 158, 56272].

[5] (a) Antonios-Mccrea, W. R.; Barsanti, P. A.; Hu, C.; Jin, X.; Martin, E. J.; Pan, Y.; Lin, X.; Pfister, K. B.; Renhowe, P. A.; Sendzik, M.; Sutton, J.; Wan, L. WO 2012101062, 2012 [Chem. Abstr. 2012, 157, 294927].

(b) Yu, H.; Li, J.; Richardson, T. E.; Bharathi, P.; Heasley, B. H.; Goutopoulos, A. WO 2013012848, 2013 [Chem. Abstr. 2013, 158, 215679].

(c) Geneste, H.; Ochse, M.; Drescher, K.; Behl, B.; Laplanche, L.; Dinges, J.; Jakob, C. WO 2013000994, 2013 [Chem. Abstr. 2013, $158,158576]$.

(d) Bodil Van Niel, M.; Fauber, B.; Gaines, S.; Gobbi, A.; Rene, O.; Vesey, D.; Ward, S. WO 2014009447, 2014 [Chem. Abstr. 2014, 160, 219224].

(e) Abeywardane, A.; Brunette, S. R.; Burke, M. J.; Kirrane, T. M., Jr.; Man, C. C.; Marshall, D. R.; Padyana, A. K.; Razavi, H.; Sibley, R.; Smith, K.; Lana, L.; Snow, R. J.; Sorcek, R. J.; Takahashi, H.; Taylor, S. J.; Turner, M. R.; Young, E. R. R.; Zhang, Q.; Zhang, 
Y.; Zindell, R. M. WO 2014014874, 2014 [Chem. Abstr. 2014, 160, 234272].

[6] Yoon, S. J.; Chung, Y. H.; Lee, C. W.; Oh, Y. S.; Kim, N. D.; Lim, J. K.; Jin, Y. H. WO 199900393, 1999 [Chem. Abstr. 1999, 130, 110281].

[7] Engel, W.; Eberlein, W.; Trummlitz, G.; Mihm, G.; Doods, H.; Mayer, N.; De Jonge, A. EP 417631, 1991 [Chem. Abstr. 1999, 115, 159188].

[8] Wuitschik, G.; Rogers-Evans, M.; Buckl, A.; Bernasconi, M.; Märki, M.; Godel, T.; Fischer, H.; Wagner, B.; Parrilla, I.; Schuler, F.; Schneider, J.; Alker, A.; Schweizer, W. B.; Müller, K.; Carreira, E. M. Angew. Chem., Int. Ed. 2008, 120, 4588.

[9] Xu, R.; Czarniecki, M.; De Man, J.; Pan, J. P.; Qiang, L.; Root, Y.; Ying, S. H.; Su, J.; Sun, X. J.; Zhang, Y. P.; Yu, T.; Zhang, Y.; Hu, T.; Chen, S. H. Tetrahedron Lett. 2011, 52, 3266.

[10] (a) Grafton, M.; Mansfield, A. C.; Fray, M. J. Tetrahedron Lett. 2010, 51, 1026.

(b) Morimoto, Y.; Achiwa, K. Chem. Pharm. Bull. 1987, 35, 3845.

(c) Ellsworth, E. L.; Sciotti, R. J.; Starr, J. T. WO 2005026165, 2005 [Chem. Abstr. 2005, 142, 336263].

(d) Wang, X. X.; Zhou, G. C. Chin. J. Synth. Chem. 2009, 17, 260 (in Chinese).

(王贤洵, 周国川, 合成化学, 2009, 17, 260.)

[11] (a) Gribble, G. W. In Comprehensive Organic Synthesis, Vol. 8, Eds.: Trost, B. M., Fleming, I., Pergamon, Oxford, 1991, p. 603.

(b) Kaiser, H. P.; Muchowski, J. M. J. Org. Chem. 1984, 49, 4203.

[12] (a) Wheland, G. W. In Advanced Organic Chemistry, 2nd ed., Wiley, J.; Sons, Inc., New York, 1949, p. 373.

(b) Brown, F. R.; Van Gulick, M. N. J. Org. Chem. 1956, 21, 1046.

[13] (a) Padwa, A.; Dent, W. J. Org. Chem. 1987, 52, 235. (b) Pandey, G.; Banerjee, P.; Gadre, R. S. Chem. Rev. 2006, 106, 4484.

[14] Bachman, G. B.; Tanner, H. A. J. Org. Chem. 1939, 4, 493.

(b) Ballesteros, P.; Roberts, B. W.; Wong, J. J. Org. Chem. 1983, 48, 3603.

(c) Ponticello, I. S. US 4056543, 1978 [Chem. Abstr. 1978, 88, 51497].

(d) De Keyser, J. L.; De Cock, C. J. C.; Poupaert, J. H.; Dumont, P. J. Org. Chem. 1988, 58, 4859.

[15] Page, P. C. B.; Gambera, G.; Hayman, C. M.; Edgar, M. Synlett 2006, 3411.

[16] Rajesh, K. M.; Cristina, M. C.; Kevin, D. R.; Edward, T. Org. Lett. 2007, 9, 575.

[17] (a) Yu, C. Z.; Liu, B.; Hu, L. Q. J. Org. Chem. 2011, 66, 5413.

(b) Zarudii, F. S.; Lazareva, D. N.; Kurmaeva, E. S.; Chalova, O. B.; Kiladze, T. K.; Kantor, E. A.; Rakhmankulov, D. L. Pharm. Chem. J. 1985, 19, 108.

(c) Tsai, F. H.; Overberger, C. G.; Zand, R. Biopolymers 1990, 30, 1039.

(d) Zhao, D. L.; Chen, C. Y.; Xu, F.; Tan, L. S.; Tillyer, R.; Pierce, E. M.; Moore, R. J. Org. Synth. 2000, 77, 12.

[18] Huang, H. M.; Liu, X. C.; Deng, J.; Qiu, M.; Zheng, Z. Org. Lett. 2006, $8,3359$.

[19] Chen, Y.; Kervio, E.; Retey, J. Helv. Chim. Acta 2002, 85, 552

[20] Morimoto, Y.; Achiwa, K. Chem. Pharm. Bull. 1987, 35, 3845.

[21] Engel, W.; Eberlein, W.; Trummlitz, G.; Mihm, G.; Doods, H.; Mayer, N.; De Jonge, A. EP 417631, 1991 [Chem. Abstr. 1991, 115, 159188].

[22] Aranha, R. M.; Bowser, A. M.; Madalengoitia, J. S. Org. Lett. 2009, 11, 575 . 